\title{
Cross Power Spectral Density between Two-Lead ECG Signals at the Termination of Obstructive Sleep Apnea with or without Arousals
}

\author{
AH Khandoker, CK Karmakar, M Palaniswami \\ The University of Melbourne, Victoria, Australia
}

\begin{abstract}
This study examined the changes of cross power spectrum density (CPSD) of ECG signals between two leads induced during OSA events and the events following its termination with/without arousals. Total 250 OSA events were selected in which only 60 events were not accompanied with ASDA arousals. Total 250 clips (5second window containing a cycle of inspiration and expiration) during OSA and 250 clips at termination of OSA events from each lead were extracted. CPSD analysis was performed between two leads ECG clips for OSA and its termination. CPSDs of the clips at all frequencies $(0 \sim 128 \mathrm{~Hz})$ were compared for OSA events and its termination with and without arousals. The results show that during OSA and its termination events without arousals, CPSDs at some frequencies are significantly $(p<0.01)$ higher than that in the events with arousals. Results could be useful for predicting the occurrence of respiratory event related cortical arousal using ECGs.
\end{abstract}

\section{Introduction}

The termination of an obstructive sleep apnea (OSA) episode is identified as airflow increase/recovery accompanying increased inspiratory effort and sympathetic cardiovascular responses to hypoxia and hypercapnia mostly leading to arousals (American sleep disorder association, ASDA) [1].

There is no clear association between the severity of OSA, as measured by the number of apneas and hypopneas per hour sleep, and the degree of daytime sleepiness [2].

A recently developed EEG analysis technique, based on neural net processing, allows a quantitative second-bysecond description of sleep "depth" [3], and thus the pattern and extent of sleep fragmentation around a respiratory event can be explored in greater detail.

However, not all apnoeas/hypopnoeas are terminated by visible cortical arousals, which are absent in about
$30 \%$ of them [4]. Also, association between arousal and upper airway opening in obstructive hypopneas-apneas, was found to be incidental [5].

The arousals may be manifested only by autonomous responses such as an increase in heart rate or blood pressure, but other features of brainstem activity may be seen, such as increase in respiratory frequency or depth and gross body or limb movements. The autonomic reaction may terminate apnoeas/hypopnoeas at thalamus/hypothalamus level [6] but they are not represented at the cortex, possibly due to the higher cortical arousal threshold. We hypothesize that quantifiable alterations occurs in ECG signals of multiple leads during OSA event and its termination when no visible arousal is detected.

Therefore, the aim of this study was to analyze ECG signals during the OSA events and OSA termination events with or without visible EEG arousals by cross power spectral density analysis.

\section{.2. Methods}

\subsection{Subjects and sleep studies}

The polysomnograms of 7 OSAS patients (OSAS + ), (mean \pm SD) age $50 \pm 9$ yrs, body mass index (BMI) $29 \pm 2$ $\mathrm{kg} / \mathrm{m} 2$, were analysed. All subjects were free of any cardiac history. Diagnosis was based on clinical symptoms and polysomnographic (PSG) outcomes. PSG study included electroencephalogram (channel C3A2/C4-A1; sampling frequency $=256 \mathrm{~Hz}$ ), left and right electrooclugram, leg movements, body positions, thoracic and abdominal wall expansion (by respiratory inductive plethysmography), oronasal airflow (by Nasal pressure, Pnasal), arterial oxygen saturation $\mathrm{SaO} 2$ (by pulse oximetry), submental electromyography (EMG) and ECG (sampling frequency $=256 \mathrm{~Hz}$ with a resolution of 16bits/sample). Respiratory events were scored using criteria proposed by the AASM[7-8]. In OSAS patients, OSA was defined as the complete cessation of breathing 
for $>10 \mathrm{sec}$ associated with a larger decrease than $3 \%$ in arterial $\mathrm{O}_{2}$ saturation or an arousal. Apneas are all obstructive. In this study, apnea hypopnea index (AHI) $>10$ was chosen as the threshold to identify the presence of OSAS + and $\mathrm{AHI}<5$ for OSAS-.

\subsection{Cross power spectral density analysis}

Total 250 OSA events were selected in which only 60 events were not accompanied with ASDA arousals [1]. Among 250 OSA events, 250 clips (5-second window containing a cycle of inspiration and expiration) during OSA and 250 clips at termination of OSA events from each lead were extracted. An arousal was scored when an abrupt shift to a faster EEG frequency (including theta, alpha and /or greater frequencies but no spindles) occurred and lasted 3-15 sec which was based on ASDA definition [1]. Cross power spectral density which is the distribution of power per unit frequency[9], between two lead ECG signals for OSA and termination events with/without arousals over the frequency band (0-128 $\mathrm{Hz}$ ) was obtained CPSDs of the two lead ECG signals were estimated using the Welch's averaged modified periodogram method of spectral estimation.

\section{Results}

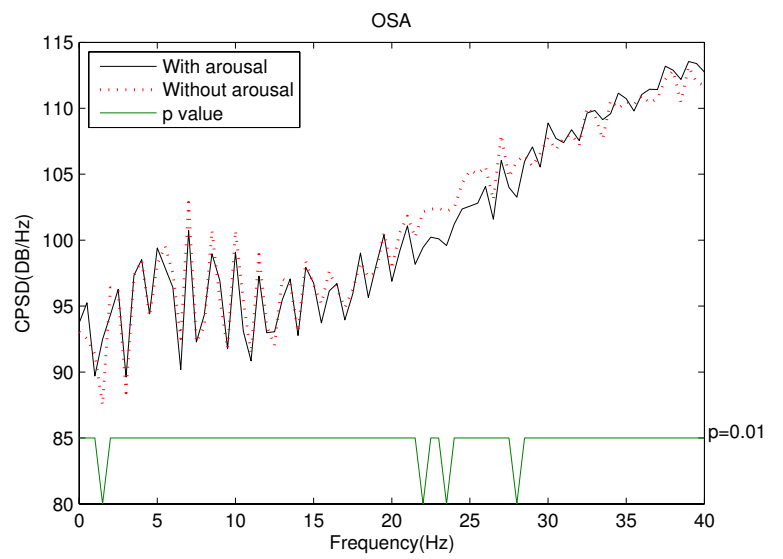

Figure 1. Mean cross power spectral density (CPSD) of two leads ECG signals (5 seconds) during OSA events with and without arousals. CPSDs are not statistically different after $40 \mathrm{~Hz}$.



Figure 2. Mean cross power spectral density (CPSD) of two leads ECG signals (5 seconds) during OSA termination events with and without arousals.

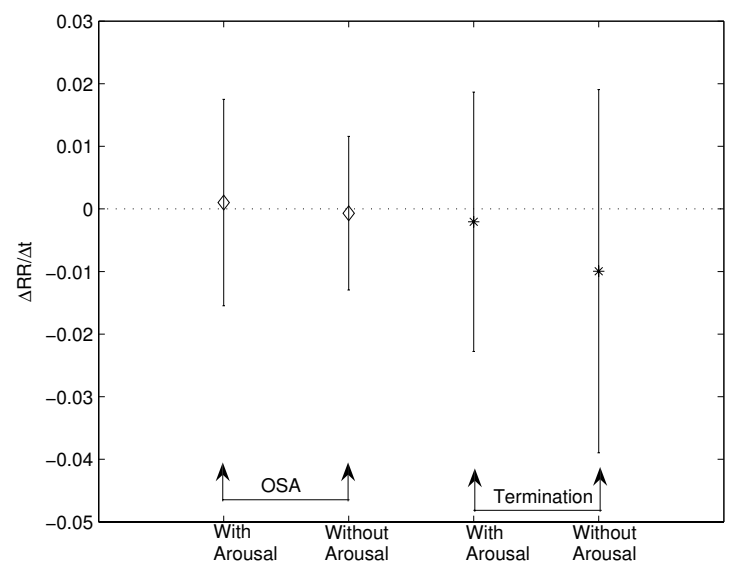

Figure 3. Mean and SD values of first derivatives of RR intervals in the middle 5 seconds of OSA events and 5 seconds termination events with and without arousals.

\section{Discussion and conclusions}

CPSDs of two lead ECGs is the DFT of crosscorrelations between the two lead ECGs. CPSDs in the higher frequencies are found to be higher as seen in Figures 1\&2. During OSA events, CPSDs at 1.5, 22 and $23.5 \mathrm{~Hz}$ are significantly $(\mathrm{p}<0.01)$ higher in the events without arousals than that in the events with arousals. Similarly, during OSA termination events, CPSDs at 3, 4, $4.5,21,22$ and $23.5 \mathrm{~Hz}$ are significantly $(\mathrm{p}<0.01)$ higher in the events without arousals than that in the events with arousals. In ECG signal, power in the frequencies higher than $20 \mathrm{~Hz}$ reflect EMG from respiratory muscles activity 
[10]. We speculate that intensity of respiratory effort during OSA events accompanied with arousals may be different than that in the events without arousals.

Even the mildest hypopnea would result in severe desaturation unless some compensatory mechanism is engaged to restore ventilation. One very important compensatory mechanism is arousal from sleep. Arousal removes the precipitating cause of obstruction and is associated with a substantial increase in dilator activity, thereby restoring ventilation.

Recently, Younes proposed that most obstructive sleep apnea (OSA) patients could open their airway without arousal [5]. In many obstructive events, airway opening preceded arousal, and the temporal relation between arousal and airway opening suggested an incidental association [5]. Upper airway opening occurred at the same time regardless of whether arousal occurred before or after opening or did not occur at all [5]. Younes postulated that chemical drive must increase a threshold amount before the pharyngeal dilator muscles can reflexly open the airway (henceforth called effective recruitment threshold; TER).

In addition to CPSD analysis, changes in heart rate response to OSA with and without arousals have been examined. Positive rate of changes in RR intervals in the middle of OSA with arousals as shown in Figure 3 indicate that heart rate is decreasing in the middle of the events. In contrast, negative rate of the same in the OSA events without arousals suggest that heart rate is increasing in the middle of those events after reaching its minimum. Similarly, heart rate during OSA termination events without arousals shows higher rate of increasing than the events with arousals. However these results are statistically not significant.

Mathematical model for the interaction and crosscorrelation between ECG signals and respiratory events with or without arousal should be attempted in future. Present detection algorithm for Respiratory event related arousals using EEG and EMG could be supplemented with ECG.

We conclude that cross correlations of two lead ECG signals during OSA events with or without arousals are different at some frequencies.

\section{Acknowledgements}

This study was supported by an Australian Research Council (ARC) Linkage Project with Compumedics Pty Ltd (LP0454378). The authors would like to thank all members of research and innovation team of
Compumedics for providing sleep studies and their valuable advices, feedback and support.

\section{References}

[1] American Sleep Disorders Association (ASDA) Task Force Report. EEG arousals: scoring rules and examples. Sleep 1992; 15:173-184.

[2] Stradling JR, Pitson DJ, Bennett L, Barbour C, Davies RJO. Variation in the arousal pattern after obstructive events in obstructive sleep apnea. Am J Respir Crit Care Med 1999;159:130-136.

[3] Pardey J, Roberts S, Tarassenko L, and Stradling J. A new approach to the analysis of the human sleep-wakefulness continuum. J. Sleep Res. 1996:5:201-210.

[4] Rees K, Spence DP, Earis JE, Carverley PM. Arousal responses from apnoeic events during NREM sleep. Am J Respir Crit Care Med 1995; 152: 1016-1021

[5] Younes M. Role of Arousals in the Pathogenesis of Obstructive Sleep Apnea. Am J Respir Crit Care Med 2004: 169; 623-633.

[6] Martin SE, Wraith PK, Deary IJ, Douglas NJ. The effect of non-visible sleep fragmentation on daytime function. Am J Respir Crit Care Med 1997; 155: 1596-1601

[7] AMERICAN ACADEMY OF SLEEP MEDICINE (AASM) TASK FORCE. Sleep-related breathing disorders in adults: recommendations for syndrome definition and measurement techniques in clinical research. Sleep 1999; 22:667-689.

[8] Rechtschaffen A and Kales A, eds. A Manual of Standardized Techniques and Scoring Systems for Sleep Stages of Human Subjects. National Institute of Health Washington: US govt printing press, 1968:204.

[9] Rabiner, L.R., and B. Gold. Theory and Application of Digital Signal Processing, Englewood Cliffs, NJ: PrenticeHall, 1975. Pgs.414-419.

[10] Thakor NV, Webster JG, Tompkins WJ. Estimation of QRS ComplexPower Spectra for Design of a QRS Filter. IEEE Transactions on Biomedical Engineering 1984, 31(11):702-706

Address for correspondence

Ahsan Khandoker

Dept. of Electrical \& Electronic Engg. The University of Melbourne, VIC -3010, Australia.

E-mail: a.khandoker@ee.unimelb.edu.edu.au 